\title{
Research on Application of Database Architecture in Computer Software Production
}

\author{
Shan Jiang ${ }^{1, \text { a }}$ \\ ${ }^{1}$ Information Technology Institute, Henan University of Chinese Medicine, Zhengzhou, Henan, \\ China, 450000 \\ aemail,
}

Keywords: Research, Application, Database Architecture, Computer Software Production

\begin{abstract}
The applications of database architecture in the computer software production mainly based on the features of the database architecture, such as reliability, data capacity, easy expansion, wide applicability, easy maintenance and so on. With the development of computer technology, computer users are demanding more and more in computer software development speed, processing ability and management ability. The development ability of computer software must keep up with the trend of the times, which also makes the computer software needs database architecture. Based on this, the characteristics of the database architecture and the requirements of the computer software production are discussed, and the application of the data architecture in the computer software is discussed.
\end{abstract}

\section{Introduction}

Nowadays, the field of computer popularization and application continues to expand, and it has gradually become the most widely used high-tech products in all walks of life. It has brought great convenience to social life and production. With the rapid development of network technology, especially database indexing, network testing and engineering structure design, the application of computer software has played a vital role, the article mainly for the database architecture in the application of computer software for analysis, The application of database architecture can effectively break the shackles of traditional computer production mode, and constantly enrich the functions of computer software to improve the security and reliability of computer software for computer software defects or vulnerabilities regularly updated and optimized so that To better meet the actual needs of computer users. Therefore, the article for the computer software production on the database architecture application research has very important practical significance.

\section{The Basic Concept and the Development Stage of the Database}

The database has two meanings. In a narrow sense, the database can be regarded as the electronicization of the folder. As the storage space of the electronic file, the user can increase, delete, delete and update the data in the electronic folder. Broadly speaking, the database is to use a variety of ways to store data in a space that can be used by multiple users, data collection and application of different, and is characterized by a small redundancy.

At present, the database has undergone the manual management stage, the file system stage, database system stage and advanced database stage.

(1) The artificial management stage, the database in the manual management stage, computer technology is still in the early stages of development, computer hardware and software development are not perfect, especially software, in the period did not produce, at this stage of database management Is the use of manual management, by the programmer on the database of data records, storage and management, storage of data information hardware devices are usually tapes, tapes and cards, when the data storage device problems, the user needs to Program to rewrite.

(2) The file system stage, research and development and application of the hard drive and operating system, that is, the use of storage devices and file management system for data management, hard disk, not only to increase the amount of data stored data, occupy a relatively 
small space, In cooperation with the operating system, to create a more stable data management system, but there will be a lot of data redundancy, due to the large amount of data, the management and maintenance of relatively high difficulty.

(3) The database system stage, with the development and popularization of computer technology, computer users to gradually improve the function of the database, in order to improve database sharing capabilities and reduce data redundancy and change the logical structure of data, Computer program, based on the only lower maintenance costs, at this stage of the development of a large number of database products, and database management level has also been significantly improved.

(4) The advanced database stage, that is, the rapid development of computer technology and network technology, the previous database system cannot meet the actual needs of computer users, such as semi-structural database model, relational database model , Hierarchical database model and mesh database model. At the same time, with the application of various new technologies, such as data stream technology and data mining technology, it can provide a reliable reference for the computer software.

\section{The Computer Software Analysis}

With the rapid development of computer technology, its popularity continues to expand, the number of computer users continues to increase and the computer software requirements continue to increase. From a technical point of view, the computer software refers to the user using a variety of programming language and computer programming language synthesis of the corresponding program, because the division of computer software is very strict, the kind of collection requirements and order is relatively high. When users need some kind of computer software, you can find and download through the network to meet their needs computer software, such as playback software, office software and chatting software.

However, the difficulty of making computer software is relatively high, in the development process need to consider many aspects, including: First, the production of computer software, should ensure that the computer software has a certain value, and analyze the feasibility of computer software production Second, in the production of computer software, can not appear in violation of relevant laws and regulations of the phenomenon, for example, a number of lawless elements making virus software, invasive software, and so on, so that the computer software cannot be used in the production of computer software, For the theft of information from other users, the destruction of computer networks, such acts violate the relevant laws, will be subject to legal sanctions, therefore, in the production of computer software, should ensure that the green software and security; When making computer software, developers need to collect a lot of data information, or software will be completed after the completion of a number of loopholes, delayed a lot of time and spend a lot of manpower, financial production of computer software, not only cannot meet the actual needs of users, Will give users a certain amount of damage. Thus, in the production of computer software, we should consider the above factors, in order to ensure the feasibility of computer software production, in line with relevant laws and regulations, and reference a large number of data, based on this to provide users with the actual needs of their computer software.

\section{The Software Design and Analysis of Database System}

The development quality of database system design plays an important role in the reform and innovation of computer technology, in large part, the database system is an indispensable part of the computer software system. Therefore, the database system design should base on the structure and establishment of the database and take software engineering technology as a support to design. The establishment of the database system, it is necessary for some language development technology to the way the language program to write some of the practical procedures and functional modules for the design and development, which is very important and necessary. From this, we can also find more complex system software through the use of software engineering technology $\mathrm{R} \& \mathrm{D}$ design, and to achieve a higher level of standard R \& D quality. 


\section{The Application Analysis of Database Architecture in the Computer Software Production}

Achieve the Model and Structure of the Database Creation. At this stage, the computer users to computer software function needs continue to improve, if a computer software has only one function, you cannot meet the actual needs of computer users to WPS, for example, WPS software features include PPT slides, EXCEL Tables and WORD documents, and software systems will be regularly updated and optimized to meet the actual needs of modern office, and also can effectively improve work efficiency, reduce the intensity of work and reduce the amount of tasks, more and more Office staff of all ages, and gradually become the most widely used at this stage one of the office software. The document, for example, at this stage of the structure and mode of many documents, paragraph layout, fonts can be adjusted, especially the chapter, view, review, insert and other functions, can effectively improve office efficiency, intelligent, automated office, Get rid of the shackles of traditional manual office. Therefore, the application of database architecture in computer software production, to achieve the database creation of the model and structure and with the expansion and optimization of the database architecture, computer software can provide a richer feature to better meet the computer users the need for software functionality.

The Table Information Processing. Database architecture in the field of computer software production applications make computer software production work more efficient, better quality. For example, in the production of computer software, through the use of the database structure of the table information processing functions, a large number of data can be automated information input and analysis. Compared with the traditional manual recording and processing methods, not only more efficient, but also to avoid the error caused by human errors record problems. When the database is created, the user can view and recall the information in the database at any time, and can also store the information separately such a table can be used as a database. When the computer users in the database information retrieval, but also according to their actual needs to create the appropriate list, the model of information in the database assessment, observation. Therefore, in the production of computer software, you should make full use of the form of information processing, the required data collection, analysis and collation for computer software production to provide a reliable and effective reference to ensure that the creation of computer software functions to meet the user's Actual demand.

Provide A Reference for the Computer Software Updates. All the computer software is not perfect, with the computer software application time increases, the software in the application process more or less there will be some problems or loopholes, through the use of database architecture, computer software can provide information on the existence of the problems or vulnerabilities in a timely manner to repair, in order to ensure that computer software functions to meet user needs. For example, QQ chat software, QQ as the most popular chat software at this stage, the database structure to the user's operation and feedback information collection, and this information back to the developers, developers based on the above information QQ chat software updates and Optimization, to solve the problems which exist, to provide users with more secure, convenient chat environment, in order to obtain more users of all ages. Database architecture on the computer, it is a very important aspect. In order to prevent such problems, it is necessary to make effective intervention on the core server, so that the intervening of the core server can make it possible to solve the problems of the core server. To deal with a variety of demands, and the face of the risk of downtime, the need for the core processor to deal with information and maintain an orderly record operation, so that customers can query data, registration and order operations to preserve information to meet the database Query and management needs.

\section{Conclusion}

In today's world, it is an era of rapid economic development and rapid development of science and technology. In the new century, computers play an increasingly important role in people's daily lives. Network provides the most convenient way to people's production and life and it has brought unprecedented convenience, especially the clever combination of computer and database 
application has brought unprecedented opportunities and convenience to people's production and life. The application of the network is a comprehensive subject, which covers the technology, management, system use, logic strategy and so on. Database is a huge data system with rich content, in which the system also covers a lot of scientific and technological information and practical value. I believe that after the rapid development of the network and communication in the future, the computer database will get greater research space and more guidance and get more application in more practice areas. Computer database update will develop with the development of science and technology and network, and the phenomenon reflected in the data will be more and more. Better study of the database architecture and better development of Internet media are the most important topic in the production and application of computer database architecture.

\section{References}

[1] Ting Li. Science \& Technology and Law, Vol. 6 (2014) No 53, p.63-66

[2] Mingde Li. Information and Computers, Vol. 12 (2015) No 27, p.25-27

[3] Binbo She. Computer Engineering, Vol. 30 (2014) No 19, p.144-145

[4] Yan Hong. Computer Knowledge and Technology, Vol. 29 (2008) No 27, p.52-55

[5] Yurui Zhang. Information and Computers, Vol. 8 (2013) No 27, p.57-60 